\title{
Effects of case volume and comprehensive stroke center capabilities on patient outcomes of clipping and coiling for subarachnoid hemorrhage
}

\author{
Ryota Kurogi, MD, PhD, ${ }^{1}$ Akiko Kada, $\mathrm{MPH},{ }^{2}$ Kuniaki Ogasawara, MD, $\mathrm{PhD},{ }^{3}$ \\ Takanari Kitazono, MD, PhD, ${ }^{4}$ Nobuyuki Sakai, MD, PhD, ${ }^{5}$ Yoichiro Hashimoto, MD, PhD, ${ }^{6}$ \\ Yoshiaki Shiokawa, MD, PhD, ${ }^{7}$ Shigeru Miyachi, MD, PhD, ${ }^{8}$ Yuji Matsumaru, MD, PhD, ${ }^{9}$ \\ Toru Iwama, MD, PhD, ${ }^{10}$ Teiji Tominaga, MD, PhD, ${ }^{11}$ Daisuke Onozuka, PhD,12 \\ Ataru Nishimura, MD, PhD, ${ }^{1}$ Koichi Arimura, MD, PhD, ${ }^{1}$ Ai Kurogi, MD, ${ }^{1}$ Nice Ren, MD, ${ }^{1}$ \\ Akihito Hagihara, DMSc, MPH, ${ }^{12}$ Yuriko Nakaoku, MD, ${ }^{12}$ Hajime Arai, MD, PhD, ${ }^{13}$ \\ Susumu Miyamoto, MD, PhD, ${ }^{14}$ Kunihiro Nishimura, MD, PhD, ${ }^{12}$ and Koji lihara, MD, PhD, ${ }^{1}$ \\ on behalf of the J-ASPECT Study Collaborators
}

\begin{abstract}
${ }^{1}$ Department of Neurosurgery, Graduate School of Medical Sciences, Kyushu University, Fukuoka; ${ }^{2}$ Department of Clinical Trials and Research, National Hospital Organization, Nagoya Medical Center, Nagoya; ${ }^{3}$ Department of Neurosurgery, Iwate Medical University, Morioka; ${ }^{4}$ Department of Medicine and Clinical Science, Graduate School of Medical Sciences, Kyushu University, Fukuoka; ${ }^{5}$ Department of Neurosurgery, Kobe City Medical Centre General Hospital, Kobe; ${ }^{6}$ Department of Neurology, Kumamoto City Hospital, Kumamoto; ' ${ }^{D}$ epartment of Neurosurgery, Kyorin University School of Medicine, Mitaka; ${ }^{8}$ Department of Neurosurgery, Aichi Medical University, Nagakute; ' Department of Neurosurgery, University of Tsukuba, Tsukuba; ${ }^{10}$ Department of Neurosurgery, Gifu University Graduate School of Medicine, Gifu; ${ }^{11}$ Department of Neurosurgery, Tohoku University School of Medicine, Sendai; ${ }^{12}$ Department of Preventive Medicine and Epidemiology, National Cerebral and Cardiovascular Center, Suita; ${ }^{13}$ Department of Neurosurgery, Juntendo University School of Medicine, Tokyo; and ${ }^{14}$ Department of Neurosurgery, Kyoto University Graduate School of Medicine, Kyoto, Japan
\end{abstract}

OBJECTIVE Improved outcomes in patients with subarachnoid hemorrhage (SAH) treated at high-volume centers have been reported. The authors sought to examine whether hospital case volume and comprehensive stroke center (CSC) capabilities affect outcomes in patients treated with clipping or coiling for $\mathrm{SAH}$.

METHODS The authors conducted a nationwide retrospective cohort study in 27,490 SAH patients who underwent clipping or coiling in 621 institutions between 2010 and 2015 and whose data were collected from the Japanese nationwide J-ASPECT Diagnosis Procedure Combination database. The CSC capabilities of each hospital were assessed by use of a validated scoring system based on answers to a previously reported 25-item questionnaire (CSC score 1-25 points). Hospitals were classified into quartiles based on CSC scores and case volumes of clipping or coiling for SAH.

RESULTS Overall, the absolute risk reductions associated with high versus low case volumes and high versus low CSC scores were relatively small. Nevertheless, in patients who underwent clipping, a high case volume (> 14 cases/yr) was significantly associated with reduced in-hospital mortality (Q1 as control, Q4 OR 0.71, 95\% Cl 0.55-0.90) but not with short-term poor outcome. In patients who underwent coiling, a high case volume (> 9 cases/yr) was associated with reduced in-hospital mortality (Q4 OR 0.69, 95\% Cl 0.53-0.90) and short-term poor outcomes (Q3 [> 5 cases/yr] OR 0.75, $95 \% \mathrm{Cl} 0.59-0.96$ vs Q4 OR 0.65, 95\% Cl 0.51-0.82). A high CSC score (> 19 points) was significantly associated with reduced in-hospital mortality for clipping (OR $0.68,95 \% \mathrm{Cl} 0.54-0.86)$ but not coiling treatment. There was no association between CSC capabilities and short-term poor outcomes.

ABBREVIATIONS BAC = Brain Attack Coalition; $\mathrm{CCI}=$ Charlson Comorbidity Index; $\mathrm{CSC}=$ comprehensive stroke center; $\mathrm{DPC}=$ Diagnosis Procedure Combination; $\mathrm{JCS}=$ Japan Coma Scale; $m R S$ = modified Rankin Scale; $Q$ = quartile; $S A H=$ subarachnoid hemorrhage; WFNS = World Federation of Neurosurgical Societies.

SUBMITTED September 19, 2019. ACCEPTED December 30, 2019.

INCLUDE WHEN CITING Published online March 13, 2020; DOI: 10.3171/2019.12.JNS192584. 
CONCLUSIONS The effects of case volume and CSC capabilities on in-hospital mortality and short-term functional outcomes in SAH patients differed between patients undergoing clipping and those undergoing coiling. In the modern endovascular era, better outcomes of clipping may be achieved in facilities with high CSC capabilities.

https://thejns.org/doi/abs/10.3171/2019.12.JNS192584

KEYWORDS subarachnoid hemorrhage; clipping; coiling; case volume; comprehensive stroke center; vascular disorders

I $\mathrm{N}$ patients with subarachnoid hemorrhage (SAH), improved outcomes at high-volume centers have been reported, ${ }^{2-4,7,9,22,24,28,32,34}$ and this finding may be related to increased experience with microsurgical/endovascular procedures, perioperative management, and low failureto-rescue rates after medical and surgical complications. ${ }^{32}$

Recently, there has been increasing focus on defining the quality and value of healthcare by reporting process and outcome measures. Consequently, the Brain Attack Coalition (BAC) proposed the establishment of comprehensive stroke centers (CSCs) to care for complex stroke patients, including SAH patients. ${ }^{1,23}$ In the US, CSCs were reported to be associated with improved survival in $\mathrm{SAH}$ patients. $^{27}$

In 2010, we launched the J-ASPECT Study, a nationwide survey of acute stroke care capacity, for the proper designation of CSCs in Japan. The J-ASPECT stroke database is a hospital-based, countrywide stroke registry formed in collaboration with the Japan Neurosurgical Society and the Japan Stroke Society. ${ }^{12}$ In a previous study, we demonstrated that the CSC capabilities of a facility were associated with reduced in-hospital mortality rates of ischemic and hemorrhagic stroke patients. ${ }^{13,17}$ Hence, there is a growing consensus toward regionalizing the management of SAH and other stroke types due to the better outcomes seen in patients receiving multidisciplinary care. In this modern endovascular era, temporary trends in the increasing use of coiling and improved outcomes in SAH patients have been noted worldwide, especially in high-volume centers. However, to our knowledge there have been no previously reported studies investigating whether case volume or CSC capabilities affect the outcomes of clipping or coiling in SAH patients. In this study, we sought to examine the effects of case volume and CSC capability on patient outcomes of clipping compared with those of coiling for $\mathrm{SAH}$.

\section{Methods \\ Hospital and Patient Selection}

Hospital participation in the J-ASPECT Study was voluntary. Of the 1369 training institutions certified by the Japan Neurosurgical Society, the Japanese Society of Neurology, and the Japan Stroke Society, 621 agreed to participate in this study. The J-ASPECT Study group analyzed the Diagnosis Procedure Combination (DPC) database to gain new clinical insights into ischemic and hemorrhagic stroke, ${ }^{13,17-19}$ an approach we applied again for this cross-sectional survey. The details of the DPC database are shown in Supplementary eAppendix 1. 14,30

We identified patients hospitalized for SAH due to a ruptured aneurysm in the de-identified discharge database using the ICD-10 diagnosis codes related to SAH (I60.09). We further selected patients who had been urgently hospitalized between April 1, 2010, and March 31, 2015, and underwent SAH treatment by clipping or coiling (Fig. 1). We then extracted data on age; sex; comorbidities on admission, including those based on Charlson Comorbidity Index (CCI) scores; ${ }^{6}$ level of consciousness on admission, according to the Japan Coma Scale (JCS) (Supplementary eAppendix 2): ${ }^{13,17-19}$ and patient transfer status (transferred from another hospital). Data on patient transfer status were available only between April 1, 2013, and March 31, 2015. To maximize the accuracy of the DPC data in the mixedcase patient classification system, at least one responsible physician (e.g., physicians in charge or residents) was required to record the information from each patient's medical chart regarding diagnoses and therapies received. ${ }^{38}$

This study was approved by the Kyushu University Institutional Review Board (28-335), which waived the requirement for individual informed consent. We have documented the data, methods, and materials used to conduct the research in this report. The individual patient data are not publicly available because of the memorandum signed by the director of the participating hospitals and the principal investigator of the J-ASPECT Study group.

\section{Assessment of CSC Capabilities Using Institutional Survey}

The CSC capabilities of the participating hospitals were assessed using a validated scoring system (CSC score) in 2010. We sent out questionnaires with 25 items, as recommended by the BAC to assess CSC capabilities of facilities to train institutions of the Japan Neurosurgical Society and the Japan Stroke Society that participated in the JASPECT Study. The analysis was performed by combining the DPC data of SAH patients admitted between 2010 and 2015 with the CSC score of each participant hospital. The questionnaire items were classified into 5 categories as follows: personnel, diagnostic, specific expertise, infrastructure, and education (Supplementary eAppendix 3). ${ }^{13,14}$ One point was assigned to each item, yielding a maximum CSC score of 25. The content, construct, and predictive validity of this scoring system have been previously reported. ${ }^{18}$ Among the 621 institutions that participated in 


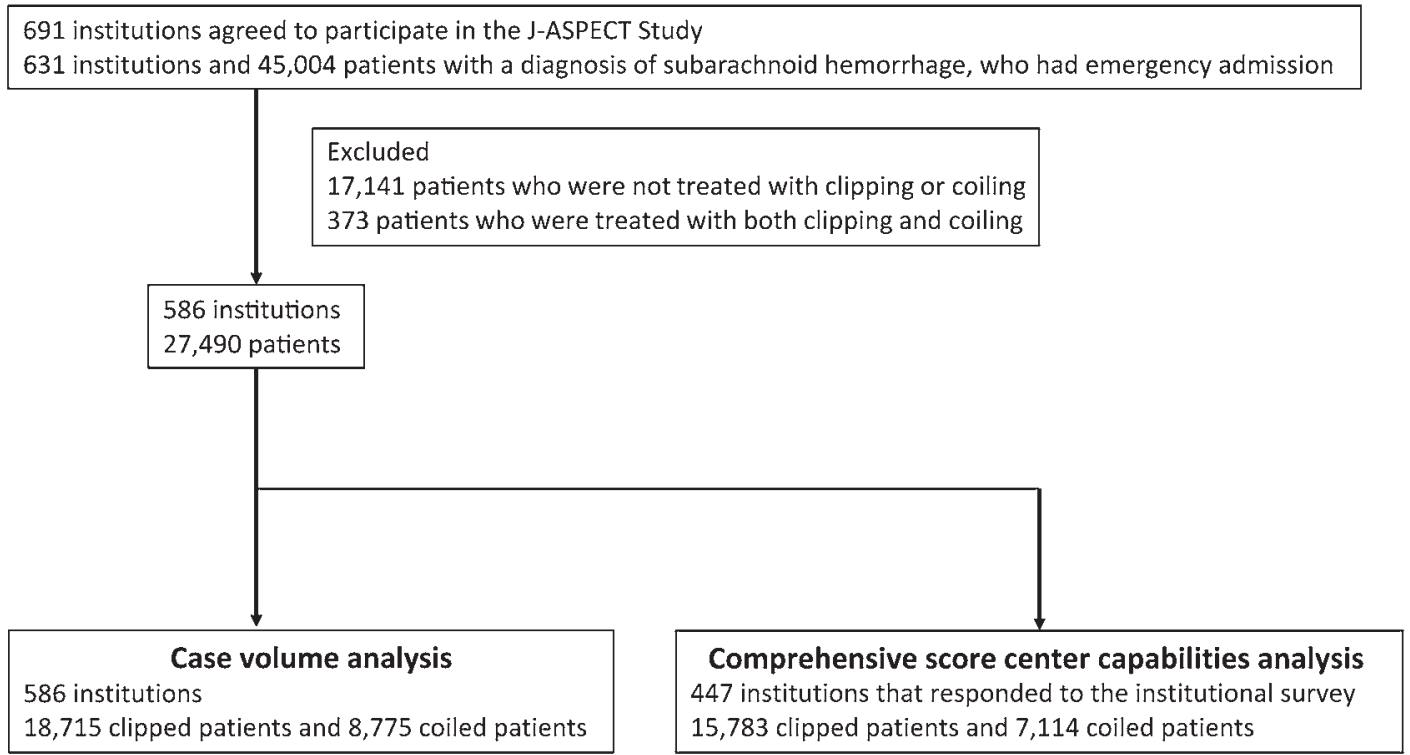

FIG. 1. Flowchart of institution and patient selection.

the J-ASPECT Study, a CSC score was assigned to each of the 447 hospitals that responded to our questionnaire.

\section{Statistical Analysis}

For statistical analysis, the modified Rankin Scale (mRS) score at discharge was dichotomized into 0-2 (good functional outcome) and 3-6 (poor functional outcome). The JCS score was treated as a categorical variable of either $0,1,2$, or 3 . The hospitals were classified into quartiles $(\mathrm{Q} 1-\mathrm{Q} 4)$ based on the case volume per year of clipping or coiling for SAH and the CSC score. The median (range) values per year for Q1, Q2, Q3, and Q4 for the clipped aneurysm patients were 3 (1-4), 6 (5-7), 10 (8-13), and 18 (14-56), respectively, for case volumes and 12 (613), 15 (14-16), 18 (17-18), and 20 (19-24), respectively, for CSC scores, and for the coiled aneurysm patients were 1 (1-2), 3 (3-4), 6 (5-8), and 12 (9-37), respectively, for case volumes and 12 (8-13), 15 (14-16), 18 (17-18), and 20 (19-24), respectively, for CSC scores. We excluded institutions with a case volume of zero.

Univariate analysis was performed with the chi-square, Kruskal-Wallis, Fisher, and Wilcoxon tests. The Kendall tau-b correlation coefficient was used to assess the strength of dependence between case volume and CSC score in the clipping and coiling groups. We used hierarchical logistic regression models to examine the associations between the case volumes of each modality and the CSC capabilities and in-hospital patient outcomes. The model had 2 levels (hospital and patient) and also employed the random effects of hospital variation and the fixed effects of CSC score and patient-related factors (age, sex, JCS score, and fiscal year of hospitalization). The lowest case volume and CSC score quartile (Q1) were used as reference values. For sensitivity analysis, the highest case volume and CSC score (Q4) were also used as references. Analyses were performed using SAS version 9.3 (SAS Institute Inc.), STATA version 12 (STATA Corp.), and SPSS version 12 (SPSS Inc.), and p values $<0.05$ were considered statistically significant.

\section{Results}

\section{Associations Between Case Volumes and Patient Charac- teristics or Outcomes}

A total of $18,715(68.3 \%)$ clipped and 8775 (31.7\%) coiled aneurysm patients were included in the analyses. Patient demographic and outcome data based on hospital case volume are shown in Tables 1 and 2.

Among the clipped aneurysm patients, the overall inhospital mortality and short-term poor outcomes (discharge mRS score 3-6) were $8.2 \%$ and $43.8 \%$, respectively. A high hospital case volume was significantly associated with reduced in-hospital mortality (e.g., Q1 9.5\% vs Q4 7.3\%) but not short-term poor outcomes.

In coiled aneurysm patients, overall in-hospital mortality and short-term poor outcomes were $13.6 \%$ and $47.5 \%$, respectively. A high case volume was significantly associated with reduced in-hospital mortality (e.g., Q1 $17.9 \%$ vs Q4 12.5\%) and short-term poor outcomes (e.g., Q1 54.8\% vs Q4 46.1\%).

\section{Associations Between CSC Score and Patient Characteristics or Outcomes}

A total of 15,783 clipped aneurysm patients and 7114 coiled aneurysm patients were included in this analysis. Patient demographics and outcomes in the clipped and coiled aneurysm patients based on hospital case volume are shown in Tables 3 and 4.

In the clipped aneurysm patients, the overall in-hospital mortality and short-term poor outcomes were $8.1 \%$ and $44.0 \%$, respectively. A high CSC score was significantly associated with reduced in-hospital mortality (e.g., Q1 $8.5 \%$ vs Q4 6.7\%) but not with reduced short-term poor outcomes. 
TABLE 1. Baseline and outcome data of clipped aneurysm patients by hospital case volume quartiles

\begin{tabular}{|c|c|c|c|c|c|c|}
\hline & Total & Q1 & Q2 & Q3 & Q4 & p Value* \\
\hline Case vol/yr, median (range) & $8(1-56)$ & $3(1-4)$ & $6(5-7)$ & $10(8-13)$ & $18(14-56)$ & \\
\hline No. of hospitals & 578 & 148 & 141 & 148 & 141 & \\
\hline No. of patients & 18,715 & 1196 & 2031 & 4778 & 10,710 & \\
\hline \multicolumn{7}{|l|}{ Baseline patient data } \\
\hline Age, yrs & $62.3 \pm 13.8$ & $61.9 \pm 13.9$ & $62.4 \pm 13.2$ & $62.3 \pm 13.9$ & $62.3 \pm 13.9$ & 0.932 \\
\hline Female & $13,032(69.6)$ & $836(69.9)$ & $1428(70.3)$ & 3328 (69.7) & $7440(69.5)$ & 0.892 \\
\hline Hypertension & $10,541(56.3)$ & $646(54.1)$ & $1172(57.7)$ & $2727(57.1)$ & $5996(56.0)$ & 0.124 \\
\hline Diabetes mellitus & $1786(9.5)$ & $89(7.4)$ & $187(9.2)$ & $466(9.8)$ & $1044(9.8)$ & 0.067 \\
\hline Dyslipidemia & $2946(15.7)$ & $149(12.5)$ & $266(13.1)$ & $765(16.0)$ & $1766(16.5)$ & $<0.001$ \\
\hline $\mathrm{CCl}$ score & $4.0 \pm 1.0$ & $4.0 \pm 1.5$ & $4.1 \pm 1.5$ & $4.0 \pm 1.5$ & $4.0 \pm 1.5$ & 0.471 \\
\hline JCS score on admission & & & & & & 0.092 \\
\hline 0 & $4453(23.8)$ & $321(26.8)$ & $520(25.6)$ & $1144(23.9)$ & $2468(23.0)$ & \\
\hline 1 & $5563(29.7)$ & $347(29.0)$ & $552(27.2)$ & $1383(28.9)$ & $3281(30.6)$ & \\
\hline 2 & $4199(22.4)$ & $260(21.7)$ & $482(23.7)$ & $1120(23.4)$ & $2337(21.8)$ & \\
\hline 3 & $4500(24.0)$ & $268(22.4)$ & $477(23.5)$ & $1131(23.7)$ & $2624(24.5)$ & \\
\hline Patient transferred to hospital $\dagger$ & $414(6.1)$ & $30(7.0)$ & $29(4.0)$ & $80(4.4)$ & $275(7.3)$ & $<0.001$ \\
\hline \multicolumn{7}{|l|}{ Patient outcome } \\
\hline In-hospital mortality & $1529(8.2)$ & $113(9.5)$ & $191(9.4)$ & $440(9.2)$ & $785(7.3)$ & $<0.001$ \\
\hline Discharge mRS score 3-6‡ & $8027(43.8)$ & $493(42.1)$ & $888(44.3)$ & $2058(43.8)$ & $4588(43.9)$ & 0.644 \\
\hline
\end{tabular}

Values are presented as number of patients (\%) or mean \pm SD unless otherwise indicated.

${ }^{*}$ Kruskal-Wallis test and chi-square test.

† Data from 2014 and 2015, $n=6742$ for clipping.

$\ddagger 395$ values missing from the clipping group.

In coiled aneurysm patients, the overall in-hospital mortality and short-term poor outcomes were $13.1 \%$ and $47.2 \%$, respectively. A high CSC score was significantly associated with reduced in-hospital mortality (e.g., Q1 12.8\% vs Q4 $12.0 \%$ ) but not with short-term poor outcomes.

\section{Multivariable Analysis: Associations Between Case Volume and Patient Outcome}

In clipped aneurysm patients, in-hospital mortality was significantly lower in the Q4 hospitals (OR 0.71, 95\% CI 0.55-0.90), whereas there was no significant difference in short-term poor outcomes based on hospital case volume. In coiled aneurysm patients, in-hospital mortality and short-term poor outcomes were significantly lower in the Q4 hospitals (OR 0.69, 95\% CI 0.53-0.90 and OR 0.65, 95\% CI 0.51-0.82, respectively). Additionally, the proportion of short-term poor outcomes was significantly lower in the Q3 and Q4 hospitals (OR 0.75, 95\% CI 0.59-0.96, and OR $0.65,95 \%$ CI $0.51-0.82$, respectively) (Table 5).

\section{Multivariable Analysis: Associations Between CSC Score and Patient Outcomes}

In clipped patients, in-hospital mortality was significantly lower in the Q4 hospitals (OR $0.68,95 \%$ CI $0.54-0.86$ ), whereas there was no significant difference in short-term poor outcomes based on hospital case volume. However, in coiled patients, there was no association between in-hospital mortality or short-term poor outcomes (discharge mRS score 3-6) and CSC capabilities (Table 6).

\section{Sensitivity Analysis}

Sensitivity analysis using Q4 as the reference demonstrated similar findings in terms of association between case volumes of clipping or coiling (Q1-Q4) and in-hospital mortality and functional outcomes of SAH patients, except for the differences in mortality for coiled aneurysm patients in case volume Q2 versus Q4 hospitals (Supplementary eAppendix 4). Similar associations were also noted between CSC score (Q1-Q4, using Q4 as reference) and in-hospital outcomes, except for the differences in mortality for coiled aneurysm patients in CSC score Q2 versus Q4 hospitals (Supplementary eAppendix 5).

\section{Correlation Between Case Volume and CSC Score in Each Patient Group}

In the clipping and coiling groups, the Kendall tau-b correlation coefficients between the case volume and CSC score were 0.23 and 0.22 , respectively.

\section{Differences Between Institutions With CSC Score and Institutions Without CSC Score}

Significantly more patients were treated with clipping in the 447 institutions with CSC scores than in the remaining 174 institutions without them. As for comorbidity, there were higher rates of hypertension and lower rates of 
TABLE 2. Baseline and outcome data of coiled aneurysm patients by by hospital case volume quartiles

\begin{tabular}{|c|c|c|c|c|c|c|}
\hline & Total & Q1 & Q2 & Q3 & Q4 & p Value* \\
\hline Case vol/yr, median (range) & $4(1-37)$ & $1(1-2)$ & $3(3-4)$ & $6(5-8)$ & $12(9-37)$ & \\
\hline No. of hospitals & 475 & 136 & 107 & 116 & 116 & \\
\hline No. of patients & 8775 & 537 & 975 & 1726 & 5537 & \\
\hline \multicolumn{7}{|l|}{ Baseline patient data } \\
\hline Age, yrs & $63.1 \pm 15.4$ & $63.3 \pm 15.4$ & $63.1 \pm 15.2$ & $63.2 \pm 15.5$ & $63.1 \pm 15.4$ & $>0.999$ \\
\hline Female & $6007(68.5)$ & $363(67.6)$ & $672(68.9)$ & $1177(68.2)$ & $3795(68.5)$ & 0.949 \\
\hline Hypertension & $4716(53.7)$ & $291(54.2)$ & $549(56.3)$ & $938(54.4)$ & 2938 (53.1) & 0.271 \\
\hline Diabetes mellitus & $691(7.9)$ & $43(8.0)$ & $79(8.1)$ & $134(7.8)$ & $435(7.9)$ & 0.99 \\
\hline Dyslipidemia & $1396(15.9)$ & $65(12.1)$ & $150(15.4)$ & $240(13.9)$ & $941(17.0)$ & 0.001 \\
\hline $\mathrm{CCl}$ score & $4.0 \pm 1.6$ & $4.1 \pm 1.6$ & $4.0 \pm 1.5$ & $4.0 \pm 1.6$ & $4.0 \pm 1.6$ & 0.968 \\
\hline JCS score on admission & & & & & & 0.636 \\
\hline 0 & $1677(19.1)$ & 97 (18.1) & $185(19.1)$ & $342(19.8)$ & $1053(19.0)$ & \\
\hline 1 & $2441(27.8)$ & $149(27.7)$ & $265(27.2)$ & $468(27.1)$ & $1559(28.2)$ & \\
\hline 2 & $1957(22.3)$ & $113(21.0)$ & $210(21.5)$ & $384(22.2)$ & $1250(22.6)$ & \\
\hline 3 & $2700(30.8)$ & $178(33.1)$ & $315(32.3)$ & $532(30.8)$ & $1675(30.3)$ & \\
\hline Patient transferred to hospital $\dagger$ & $346(9.2)$ & $2(1.3)$ & $18(4.7)$ & $47(7.9)$ & $279(10.6)$ & $<0.001$ \\
\hline \multicolumn{7}{|l|}{ Patient outcome } \\
\hline In-hospital mortality & $1196(13.6)$ & $96(17.9)$ & $137(14.1)$ & $272(15.8)$ & $691(12.5)$ & $<0.001$ \\
\hline Discharge mRS score 3-6‡ & $4080(47.5)$ & $288(54.8)$ & $469(49.5)$ & $831(48.9)$ & $2492(46.1)$ & $<0.001$ \\
\hline
\end{tabular}

Values are presented as number of patients (\%) or mean \pm SD unless otherwise indicated.

${ }^{*}$ Kruskal-Wallis test and chi-square test.

† Data from 2014 and 2015, $n=3762$ for coiling.

$\ddagger 193$ values missing from the coiling group.

dyslipidemia among SAH patients (Supplementary eAppendix 6).

\section{Multivariable Analysis: Association of Case Volume or CSC Score With Patient Outcomes}

There was a low correlation coefficient between CSC score and case volume; hence, we performed analysis after adjusting for both CSC score and case volume in addition to age, sex, and CCI and JCS scores. Except for Q4 of case volume in coiled aneurysm patients, we found similar associations to those found with other analyses after adjusting for each CSC score and case volume as variables (Supplementary eAppendix 7).

\section{Multivariable Analysis: Impact of Treatment Modality}

In the 15,783 clipped aneurysm patients and 7114 coiled aneurysm patients, the in-hospital mortality was significantly lower in the clipping group (OR 0.60, 95\% CI $0.54-0.67, \mathrm{p}<0.001$ ), whereas there was no significant difference between the 2 groups in discharge mRS score 3-6 (OR 1.02, 95\% CI 0.94-1.10, $\mathrm{p}=0.651$ ).

\section{Discussion}

Using linkage data between 2 large nationwide databases (an institutional survey and the DPC database of the J-ASPECT Study), we found that hospital case volume was significantly associated with reduced in-hospital mortality in both clipped and coiled aneurysm patients; we also found improved functional short-term outcomes in coiled aneurysm patients after adjusting for disease severity determined using the JCS score. Additionally, hospital CSC capabilities were significantly associated with reduced in-hospital mortality in clipped but not coiled aneurysm patients; there was only a weak association between case volume and CSC score in both modalities.

The technical skill and perioperative management required for the clipping and coiling procedures are different. However, most previous studies on SAH have defined the case volume as the combined number of patients undergoing clipping and coiling, or the number of admissions. $2,3,6,9,24,25,28,32,34$ We divided the case volume of SAH patients into quartiles in each modality (clipping or coiling) separately for the following reasons. First, the ratio of clipping to coiling for ruptured intracranial aneurysms differs between high- and low-volume hospitals. ${ }^{22,25}$ Additionally, the level of experience in procedural skill and perioperative management of clipping or coiling may depend on the ratio of clipping to coiling in the institution. In addition, the difference in disease severity on admission between patients treated with clipping versus coiling affects patient outcomes. ${ }^{18}$ Therefore, we believe that our study reflects the effect of case volume in each modality more precisely.

We conducted a sensitivity analysis, which shows a comparison of patient outcomes at Q4 institutions with those at Q1-Q3 institutions, to determine the point where there is no significant difference. However, our findings 
TABLE 3. Baseline and outcome data of clipped aneurysm patients by hospital CSC score quartiles

\begin{tabular}{|c|c|c|c|c|c|c|}
\hline & All & Q1 & Q2 & Q3 & Q4 & $p$ Value* \\
\hline CSC score, median (range) & $16(6-24)$ & $12(6-13)$ & $15(14-16)$ & $18(17-18)$ & $20(19-24)$ & \\
\hline No. of hospitals & 443 & 125 & 128 & 88 & 102 & \\
\hline No. of patients & 15,783 & 3188 & 3732 & 3213 & 5650 & \\
\hline \multicolumn{7}{|l|}{ Baseline patient data } \\
\hline Age, yrs & $62.2 \pm 13.8$ & $62.4 \pm 13.8$ & $62.0 \pm 13.9$ & $61.9 \pm 13.7$ & $62.4 \pm 13.6$ & 0.124 \\
\hline Female & $10,978(69.6)$ & $2238(70.2)$ & $2573(68.9)$ & $2240(69.7)$ & $3927(69.5)$ & 0.722 \\
\hline Hypertension & $8999(57.0)$ & $1944(61.0)$ & $2254(60.4)$ & $1940(60.4)$ & $2861(50.6)$ & $<0.001$ \\
\hline Diabetes mellitus & $1479(9.4)$ & $284(8.9)$ & $435(11.7)$ & $306(9.5)$ & $454(8.0)$ & $<0.001$ \\
\hline Dyslipidemia & $2458(15.6)$ & $456(14.3)$ & $593(15.9)$ & $519(16.2)$ & $890(15.8)$ & 0.161 \\
\hline $\mathrm{CCl}$ score & $4.0 \pm 1.5$ & $4.0 \pm 1.5$ & $4.1 \pm 1.6$ & $4.0 \pm 1.5$ & $4.0 \pm 1.5$ & 0.004 \\
\hline JCS score on admission & & & & & & $<0.001$ \\
\hline 0 & $3864(23.3)$ & $779(24.4)$ & $901(24.1)$ & $712(22.2)$ & $1292(22.9)$ & \\
\hline 1 & $4751(30.1)$ & 1024 (32.1) & $1142(30.6)$ & $886(27.6)$ & 1699 (30.1) & \\
\hline 2 & $3600(22.8)$ & $736(23.1)$ & $832(22.3)$ & $754(23.5)$ & $1278(22.6)$ & \\
\hline 3 & $3748(23.7)$ & $649(20.4)$ & $857(23.0)$ & $861(26.8)$ & $1381(24.4)$ & \\
\hline Patient transferred to hospital $\dagger$ & $357(6.9)$ & $52(5.0)$ & $44(3.7)$ & $74(7.0)$ & $187(9.8)$ & $<0.001$ \\
\hline \multicolumn{7}{|l|}{ Patient outcome } \\
\hline In-hospital mortality & $1274(8.1)$ & $272(8.5)$ & $349(9.4)$ & $274(8.5)$ & $379(6.7)$ & $<0.001$ \\
\hline Discharge mRS score 3-6‡ & $6817(44.0)$ & $1410(45.1)$ & $1597(43.8)$ & $1422(44.6)$ & 2388 (43.1) & 0.251 \\
\hline
\end{tabular}

were similar except for the difference between Q2 and Q4 in patients treated with coiling compared with those treated with clipping. The presence of unmeasured confounders in coiled aneurysm patients may explain this finding, but further studies are required to determine the reasons for these differences.

\section{Patient Demographics and Outcomes of Clipping and Coiling}

In this study, the patient characteristics and unadjusted outcomes after treatment with either the clipping or coiling modality were similar to those of our previously reported SAH cohort in Japan. ${ }^{18}$ As for comorbidities, trends reflecting decreased hypertension and diabetes incidence and increased dyslipidemia were generally noted in patients treated at hospitals with increased case volume and CSC capabilities. This finding may be explained by geographical factors, because hospitals with high case volumes and CSC capabilities are more likely to be located in urban areas. ${ }^{14}$ Compared with previous nationwide studies in the US and Canada, , ${ }^{8,26,31}$ SAH patients in our Japanese cohort were almost 10 years older and had lower mortality.

SAH patients treated with coiling had overall higher rates of in-hospital mortality than those treated with clipping. This finding may be attributable to a learning process of the hospital surgical team in the use of endovascular therapy for SAH, as reflected by the higher number of cases treated preferably with clipping between 2010 and 2015 in the Japanese nationwide database used in this study.

\section{Effect of Clipping and Coiling Case Volumes on In-Hospital SAH Patient Outcomes}

Numerous studies have reported improved outcomes in SAH patients treated at high-volume centers. . $^{24,7,9,22,24,28,32,34}$ On the other hand, a previous study in Norway did not find a relationship between treatment at hospitals with high case volume and long-term mortality, ${ }^{25}$ suggesting that the patient volume-outcome relationship may not be generalizable in other countries. Furthermore, definitions of high and low volume have varied among previous studies. ${ }^{2-5,7,9,10,22,24,25,28,32,34}$ In the US, to obtain CSC certification, institutions are required by the Joint Commission to manage at least 20 cases of SAH and perform at least 15 coiling and/or clipping procedures for ruptured cerebral aneurysms per year. ${ }^{1,32}$ However, Pandey et al. suggested that this threshold may be relatively low because according to their study the SAH patient volume-outcome association did not reach a plateau until the institution had managed more than $100 \mathrm{SAH}$ patients per year. ${ }^{32}$

Some reported studies have found better functional outcomes in high-volume centers, ,2,24,32,34 whereas others have not. ${ }^{5,10,22}$ Our results of better functional outcomes in hospitals with Q3 and Q4 case volumes may be attributable to patients being more likely to receive endovascular treatment for vasospasm, such as intraarterial vasodilator infusion or angioplasty. ${ }^{11}$ On the other hand, the lack of a significant influence of clipping case volume on functional outcomes may be explained by the possibility that cases with a higher level of difficulty are referred to hospi- 
TABLE 4. Baseline and outcome data of coiled aneurysm patients by hospital CSC score quartiles

\begin{tabular}{|c|c|c|c|c|c|c|}
\hline & All & Q1 & Q2 & Q3 & Q4 & $p$ Value* \\
\hline CSC score, median (range) & $16(8-24)$ & $12(8-13)$ & $15(14-16)$ & $18(17-18)$ & $20(19-24)$ & \\
\hline No. of hospitals & 367 & 76 & 108 & 84 & 99 & \\
\hline No. of patients & 7114 & 486 & 1558 & 1985 & 3085 & \\
\hline \multicolumn{7}{|l|}{ Baseline patient data } \\
\hline Age, yrs & $63.1 \pm 15.4$ & $62.2 \pm 15.7$ & $63.6 \pm 15.2$ & $62.5 \pm 15.3$ & $63.5 \pm 15.6$ & 0.051 \\
\hline Female & $4898(68.9)$ & $336(69.1)$ & $1069(68.6)$ & $1349(68.0)$ & $2144(69.5)$ & 0.707 \\
\hline Hypertension & $3878(54.5)$ & $289(59.5)$ & $844(54.2)$ & $1138(57.3)$ & $1607(52.1)$ & $<0.001$ \\
\hline Diabetes mellitus & $552(7.8)$ & $39(8.0)$ & $116(7.5)$ & $163(8.2)$ & $234(7.6)$ & 0.811 \\
\hline Dyslipidemia & $1092(15.4)$ & $80(16.5)$ & $219(14.1)$ & $339(17.1)$ & $454(14.7)$ & 0.046 \\
\hline $\mathrm{CCl}$ score & $4.0 \pm 1.6$ & $4.0 \pm 1.6$ & $4.1 \pm 1.6$ & $4.0 \pm 1.6$ & $4.0 \pm 1.6$ & 0.431 \\
\hline JCS score on admission & & & & & & 0.053 \\
\hline 0 & $1380(19.4)$ & $102(21.0)$ & $275(17.7)$ & $372(18.7)$ & $631(20.5)$ & \\
\hline 1 & $1951(27.4)$ & $140(28.8)$ & $479(30.7)$ & $520(26.2)$ & $812(26.3)$ & \\
\hline 2 & $1652(23.2)$ & $100(20.6)$ & $370(23.7)$ & $438(22.1)$ & $744(24.1)$ & \\
\hline 3 & $2131(30.0)$ & $144(27.4)$ & $113(32.0)$ & $129(31.6)$ & $50(25.8)$ & \\
\hline Patient transferred to hospital $†$ & $268(9.8)$ & $4(2.0)$ & $38(6.1)$ & $100(11.9)$ & $126(11.9)$ & $<0.001$ \\
\hline \multicolumn{7}{|l|}{ Patient outcome } \\
\hline In-hospital mortality & $932(13.1)$ & $62(12.8)$ & $239(15.3)$ & $261(13.2)$ & $370(12.0)$ & 0.017 \\
\hline Discharge mRS score 3-6‡ & $3297(47.2)$ & $215(45.1)$ & $724(47.3)$ & $932(47.6)$ & $1426(47.1)$ & 0.802 \\
\hline
\end{tabular}

tal settings with higher levels of surgical team experience, multidisciplinary approaches, and surveillance. However, in Japan, Hattori et al. reported no relationship between case volume and outcomes for clipping for SAH in cases of each World Federation of Neurosurgical Societies (WFNS) severity grade..$^{10}$ However, the overall in-hospital mortality rate in clipped SAH patients in that study $(9.0 \%)$ was similar to the rate in the present study. There are several possible reasons for differences in the former findings compared with the findings of the current study. First, Hattori et al. compared outcomes among institutions accredited by the Japan Neurosurgical Society as class A specialist training facilities (annual neurosurgical procedures $>100$ cases/yr), whereas the hospitals in our study varied widely. ${ }^{10}$ In fact, the number of clipping cases in the highest-volume center in the previous study was $>50$ cases,$^{10}$ whereas in the present study it ranged from 14 to 56 cases (Q4). Second, Hattori et al. examined the effect of case volume of clippings performed in $2003 .{ }^{10}$ Although clipping remains the mainstay treatment for SAH in Japan, ${ }^{10}$ a significant shift from clipping to coiling has been observed since then. ${ }^{35}$ Thus, our study represents the current status of the volume-outcome relationship of clipping and coiling for SAH in Japan.

As for coiling, a study of 534 cases from 29 tertiary referral centers in Japan examined the determinants of poor outcomes (mRS 3-6 at 12 months) in SAH patients when both clipping and coiling were available. ${ }^{39}$ The authors reported that the type of treatment modality and the size and location of aneurysms did not influence the outcome. In that report, high-volume centers ( $>30$ cases/yr, 6 centers) did not affect the outcomes. Taken together, this inconsistency in reported results is most likely due to selection bias in each study.

In the present study, the case volumes in the highest quartiles (Q4) for clipping (>14) and coiling (>9), which were associated with better outcomes, almost meet the US CSC criteria of the Joint Commission. ${ }^{1,32}$ Generally, SAH patients in high-volume centers are more likely to receive neurointensive care in a multidisciplinary setting. ${ }^{4}$ Greater aggressiveness and effectiveness of aneurysm treatment in high-volume centers may explain their lower mortality figures. Considering there was only a weak association between case volume and CSC capabilities for both the clipping and coiling modalities in this study, further research is required to determine the mechanisms underlying the sole influence of case volume.

In terms of risk reduction, when Q4 hospitals were compared to Q1 hospitals, the risk differences of in-hospital mortality for treatment by coiling and clipping were 5.4\% and $2.2 \%$, respectively. This result suggests that, based on case volume characteristics, there is a more substantial difference in benefits in terms of mortality risk reduction when aneurysm patients are treated endovascularly rather than surgically.

\section{Effect of CSC Capabilities on In-Hospital Outcomes of SAH Patients}

Unexpectedly, we found that CSC capabilities were as- 
TABLE 5. Associations of case volume of clipping or coiling by hospital quartile with in-hospital mortality and discharge mRS score 3-6 in patients with aneurysm rupture

\begin{tabular}{cccccccc}
\hline & \multicolumn{3}{c}{ Clipping } & & \multicolumn{3}{c}{ Coiling } \\
Case Vol Quartile & OR & $95 \% \mathrm{Cl}$ & $\mathrm{p}$ Value & & OR & $95 \% \mathrm{Cl}$ & $\mathrm{p}$ Value \\
\hline In-hospital mortality & & & & & & \\
\hline Q2 & 0.97 & $0.74-1.26$ & 0.798 & & 0.75 & $0.55-1.02$ & 0.063 \\
\hline Q3 & 0.95 & $0.75-1.22$ & 0.692 & & 0.85 & $0.64-1.12$ & 0.245 \\
\hline Q4 & 0.71 & $0.55-0.90$ & 0.005 & & 0.69 & $0.53-0.90$ & 0.006 \\
\hline Discharge mRS score 3-6 & & & & & & \\
\hline Q2 & 1.03 & $0.85-1.24$ & 0.768 & & 0.80 & $0.61-1.03$ & 0.084 \\
\hline Q3 & 1.02 & $0.86-1.22$ & 0.787 & & 0.75 & $0.59-0.96$ & 0.023 \\
\hline Q4 & 1.06 & $0.89-1.26$ & 0.516 & & 0.65 & $0.51-0.82$ & $<0.001$ \\
\hline
\end{tabular}

After adjustment for age, sex, and CCI and JCS scores. Q1 is used as a reference.

sociated with decreased in-hospital mortality in clipped, but not coiled, SAH patients. Prior studies have demonstrated that institutional factors such as access to endovascular services and teaching hospital status affect the outcomes of aneurysm treatment. ${ }^{16,20}$ Interestingly, the proportion of comatose clipped patients in hospitals with higher CSC capabilities (Q3 and Q4) was relatively higher in this study. Improvements in neurosurgical techniques, such as intraoperative angiography, neurocritical care, and neuroimaging, have allowed neurosurgeons to successfully care for patients who would have previously been considered too sick to treat. The availability of technically trained surgeons and access to facilities with endovascular surgery can also sway prognosis since these institutional factors often dictate treatment and were well represented by the components of the CSC score used in this study.

As subspecialty care becomes more centralized, the availability of both treatment modalities poses a dilemma in terms of patient selection. In both clipped and coiled patients, our study showed that the proportion of transferred patients was highest in hospitals with the highest case volume and the highest CSC capabilities. In this light, it is interesting to note the increasing trends in patient transfer in relation to treatment modality based on either case volume or CSC capabilities.

Previously reported studies on coronary artery bypass graft surgery $(\mathrm{CABG})^{40}$ and esophagectomy ${ }^{33}$ demonstrated that case volume alone is a relatively weak predictor of outcome and highlighted the need for greater availability of hospital-specific performance data such as measures of care processes. ${ }^{40}$

In this light, we showed that higher CSC capabilities, measured by the CSC score, are associated with reduced in-hospital mortality in SAH patients with or without surgical or endovascular treatment. ${ }^{13,14}$ A previous study in the US confirmed this finding. ${ }^{27}$ According to the J-ASPECT institutional survey conducted in 2010, 2014, and 2018, there were increasing trends in implementation of items related to endovascular treatment (e.g., board-certified endovascular physicians and 24-hour, 7-day endovascular coverage) and multidisciplinary care (e.g., stroke unit), leading to more widespread use of coiling in Japan. The present findings may be explained by the steep learning curve required to achieve coiling proficiency ${ }^{37}$ and the rapid development of endovascular technology. ${ }^{29}$ Further research is necessary to determine which items of the CSC

TABLE 6. Associations of CSC of clipping or coiling by hospital quartile with in-hospital mortality and discharge $\mathrm{mRS}$ score 3-6 in patients with aneurysm rupture

\begin{tabular}{cccccccc}
\hline & \multicolumn{3}{c}{ Clipping } & & \multicolumn{3}{c}{ Coiling } \\
\cline { 8 - 9 } Case Vol Quartile & OR & $95 \% \mathrm{Cl}$ & $\mathrm{p}$ Value & & OR & $95 \% \mathrm{Cl}$ & $\mathrm{p}$ Value \\
\hline In-hospital mortality & & & & & & \\
\hline Q2 & 1.1 & $0.87-1.39$ & 0.441 & & 1.27 & $0.89-1.82$ & 0.185 \\
\hline Q3 & 0.89 & $0.69-1.14$ & 0.341 & & 1.03 & $0.72-1.48$ & 0.851 \\
\hline Q4 & 0.68 & $0.54-0.86$ & 0.002 & & 0.94 & $0.67-1.33$ & 0.741 \\
\hline Discharge mRS score 3-6 & & & & & & \\
\hline Q2 & 0.94 & $0.78-1.14$ & 0.54 & & 1.08 & $0.79-1.46$ & 0.614 \\
\hline Q3 & 0.94 & $0.77-1.14$ & 0.51 & & 1.06 & $0.78-1.42$ & 0.727 \\
\hline Q4 & 0.87 & $0.72-1.01$ & 0.162 & & 1.01 & $0.76-1.35$ & 0.925 \\
\hline
\end{tabular}

After adjustment for age, sex, and CCl and JCS scores. Q1 is used as a reference. 
score specifically influence outcomes after surgical clipping for aneurysms.

\section{Study Limitations}

Our study has several limitations. First, the DPC database lacks several types of data, such as aneurysm characteristics, ${ }^{15}$ delayed cerebral ischemia, treatment of vasospasm, and cause of death. However, similar limitations pertain to all previous administrative database studies using diagnostic coding that examined the relationship between hospital case volume and mortality after aneurysm treatment. ${ }^{34}$ Moreover, unlike all but one ${ }^{34}$ of those studies, $, 3,3,7,9,22,24,25,28,32$ our study included adjustment for severity of SAH. The severity of SAH is an important factor that affects clinical outcome. ${ }^{21}$ Therefore, we think that our analysis allowed a more robust comparison of hospital volume and outcomes in SAH patients. Additionally, we used the JCS score rather than the Hunt and Hess grade or the WFNS scale to measure SAH severity. The JCS is widely used in Japan for the assessment of impaired consciousness in SAH patients, ${ }^{13,14,17-19}$ and JCS scores correlate with stroke outcomes. ${ }^{36}$ Second, inaccuracies with coding may exist within our DPC database, leading to an underestimation or overestimation of outcomes. ${ }^{41}$ However, we performed a validation study on SAH patients in our previous analysis of the J-ASPECT Study, ${ }^{18}$ and the high levels of accuracy of diagnoses, treatment modalities, and outcomes of this cohort contained in the DPC database were confirmed by a 2-stage sampling method. Third, this study has potential selection bias from voluntary involvement in the registry, with only about half of the 1369 institutions participating. We previously reported that the hospitals participating in this J-ASPECT Study, rather than nonparticipating hospitals, treated more than 10 cases of intracerebral hemorrhage and SAH per 6 months. Hence, hospitals actively working to improve stroke care were more likely to participate in this study, ${ }_{14}$ and therefore the present results may not be generalizable to nonparticipating hospitals. In addition, the results of associations between CSC score and outcomes should be interpreted with caution due to potential selection bias, since the CSC score was available in only 447 among the total 621 institutions. Furthermore, there were some significant differences in hospital and patient backgrounds, and such differences may be explained by the geographical location of the hospitals. Therefore, the results regarding associations between CSC score and outcomes should not be generalized to institutions that did not respond to our questionnaire for CSC capabilities. Additionally, the number of participating hospitals in this study is increasing yearly, and the assessment of CSC capabilities has been performed every 4 years after the period of this study. Thus, we are planning to evaluate the validity of the effects of the case volume and CSC score in SAH patients in the future. Fourth, the CSC score is a self-reported questionnaire and not the result of any formal certification process. In Japan, the official certification of PSC (primary stroke center) just began in 2019. The official certification of CSC in the immediate future will be determined by the Japan Stroke Society; therefore, the results of this study could have a significant impact on the recommended items and criteria for the des- ignation CSCs in Japan. After the official certification of the CSC score, we also plan to reassess the effect of CSC capabilities in SAH patients. Fifth, there is a limitation due to the large imbalance of clipping over coiling. Despite the increasing use of coiling in other countries, clipping remains the mainstay treatment for SAH. In fact, the relative merits of clipping and coiling for $\mathrm{SAH}$, and the ratio of clipping to coiling, were different between Japan and the US at the time of this study. ${ }^{18}$ Therefore, our results may not be generalizable to other countries, although the ratio of coiling for SAH has recently increased. ${ }^{39}$ Therefore, further studies will be needed in the future to assess the effects of the ratio of treatment modalities (clipping vs coiling) on patient outcomes.

\section{Conclusions}

The effects of case volume and CSC capabilities on inhospital mortality and short-term functional outcomes of institutions treating SAH patients differed for the treatment modalities of clipping and coiling. However, in Japan, the absolute risk reduction associated with institutional variations from high to low case volume and from high to low CSC score is relatively small. Nevertheless, higher case volume was significantly associated with lower in-hospital mortality in both clipped and coiled patients, with better functional outcome in patients treated with coiling. Additionally, the hospital CSC capabilities were significantly associated with lower in-hospital mortality in clipped, but not coiled, aneurysm patients. Hence, in this modern endovascular era, better outcomes of clipping for SAH may be achieved in facilities with higher CSC capabilities. Further research is required to determine the influence of unmeasured confounders.

\section{Acknowledgments}

Details of the J-ASPECT Study Collaborators are listed in Supplementary eAppendix 8. We thank the Japan Neurosurgical Society (Profs. Takamasa Kayama and Hajime Arai) and the Japan Stroke Society (Profs. Norihiro Suzuki and Susumu Miyamoto) for their collaboration.

The J-ASPECT Study (principal investigator: Koji Iihara) was supported by Grants-in-Aid from the Japanese Ministry of Health, Labour and Welfare and KAKENHI grants (25293314, 18H02914) from the Japan Society for the Promotion of Science. This research is partially supported by the Practical Research Project for Life-Style Related Diseases including Cardiovascular Diseases and Diabetes Mellitus managed by the Japan Agency for Medical Research and Development (17ek0210088h0001, 18ek0210088h0002).

\section{References}

1. Alberts MJ, Latchaw RE, Selman WR, Shephard T, Hadley MN, Brass LM, et al: Recommendations for comprehensive stroke centers: a consensus statement from the Brain Attack Coalition. Stroke 36:1597-1616, 2005

2. Bardach NS, Zhao S, Gress DR, Lawton MT, Johnston SC: Association between subarachnoid hemorrhage outcomes and number of cases treated at California hospitals. Stroke 33:1851-1856, 2002

3. Berman MF, Solomon RA, Mayer SA, Johnston SC, Yung PP: Impact of hospital-related factors on outcome after treatment of cerebral aneurysms. Stroke 34:2200-2207, 2003 
4. Boogaarts HD, van Amerongen MJ, de Vries J, Westert GP, Verbeek AL, Grotenhuis JA, et al: Caseload as a factor for outcome in aneurysmal subarachnoid hemorrhage: a systematic review and meta-analysis. J Neurosurg 120:605-611, 2014

5. Chang TR, Kowalski RG, Carhuapoma JR, Tamargo RJ, Naval NS: Impact of case volume on aneurysmal subarachnoid hemorrhage outcomes. J Crit Care 30:469-472, 2015

6. Charlson ME, Pompei P, Ales KL, MacKenzie CR: A new method of classifying prognostic comorbidity in longitudinal studies: development and validation. J Chronic Dis 40:373383, 1987

7. Cowan JA Jr, Dimick JB, Wainess RM, Upchurch GR Jr, Thompson BG: Outcomes after cerebral aneurysm clip occlusion in the United States: the need for evidence-based hospital referral. J Neurosurg 99:947-952, 2003

8. Cowan JA Jr, Ziewacz J, Dimick JB, Upchurch GR Jr, Thompson BG: Use of endovascular coil embolization and surgical clip occlusion for cerebral artery aneurysms. J Neurosurg 107:530-535, 2007

9. Cross DT III, Tirschwell DL, Clark MA, Tuden D, Derdeyn $\mathrm{CP}$, Moran CJ, et al: Mortality rates after subarachnoid hemorrhage: variations according to hospital case volume in 18 states. J Neurosurg 99:810-817, 2003

10. Hattori N, Katayama Y, Abe T: Case volume does not correlate with outcome after cerebral aneurysm clipping: a nationwide study in Japan. Neurol Med Chir (Tokyo) 47:95-101, 2007

11. Hayashi K, Hirao T, Sakai N, Nagata I: Current status of endovascular treatment for vasospasm following subarachnoid hemorrhage: analysis of JR-NET2. Neurol Med Chir (Tokyo) 54:107-112, 2014

12. Iihara K: Comprehensive stroke care capabilities in Japan: a neurovascular surgeon's perspective. Neurosurgery 62 (Suppl 1):107-116, 2015

13. Iihara K, Nishimura K, Kada A, Nakagawara J, Ogasawara $\mathrm{K}$, Ono J, et al: Effects of comprehensive stroke care capabilities on in-hospital mortality of patients with ischemic and hemorrhagic stroke: J-ASPECT study. PLoS One 9:e96819, 2014

14. Iihara K, Nishimura K, Kada A, Nakagawara J, Toyoda K, Ogasawara K, et al: The impact of comprehensive stroke care capacity on the hospital volume of stroke interventions: a nationwide study in Japan: J-ASPECT study. J Stroke Cerebrovasc Dis 23:1001-1018, 2014

15. Jaja BN, Lingsma H, Steyerberg EW, Schweizer TA, Thorpe KE, Macdonald RL: Neuroimaging characteristics of ruptured aneurysm as predictors of outcome after aneurysmal subarachnoid hemorrhage: pooled analyses of the SAHIT cohort. J Neurosurg 124:1703-1711, 2016

16. Johnston SC: Effect of endovascular services and hospital volume on cerebral aneurysm treatment outcomes. Stroke 31:111-117, 2000

17. Kada A, Nishimura K, Nakagawara J, Ogasawara K, Ono J, Shiokawa Y, et al: Development and validation of a score for evaluating comprehensive stroke care capabilities: J-ASPECT Study. BMC Neurol 17:46, 2017

18. Kurogi R, Kada A, Nishimura K, Kamitani S, Nishimura A, Sayama T, et al: Effect of treatment modality on in-hospital outcome in patients with subarachnoid hemorrhage: a nationwide study in Japan (J-ASPECT Study). J Neurosurg 128: $1318-1326,2018$

19. Kurogi R, Nishimura K, Nakai M, Kada A, Kamitani S, Nakagawara J, et al: Comparing intracerebral hemorrhages associated with direct oral anticoagulants or warfarin. Neurology 90:e1143-e1149, 2018

20. Lai PM, Lin N, Du R: Effect of teaching hospital status on outcome of aneurysm treatment. World Neurosurg 82:380385.e6, 2014
21. Le Roux PD, Elliott JP, Newell DW, Grady MS, Winn HR: Predicting outcome in poor-grade patients with subarachnoid hemorrhage: a retrospective review of 159 aggressively managed cases. J Neurosurg 85:39-49, 1996

22. Leake CB, Brinjikji W, Kallmes DF, Cloft HJ: Increasing treatment of ruptured cerebral aneurysms at high-volume centers in the United States. J Neurosurg 115:1179-1183, 2011

23. Leifer D, Bravata DM, Connors JJ III, Hinchey JA, Jauch EC, Johnston SC, et al: Metrics for measuring quality of care in comprehensive stroke centers: detailed follow-up to Brain Attack Coalition comprehensive stroke center recommendations: a statement for healthcare professionals from the American Heart Association/American Stroke Association. Stroke 42:849-877, 2011

24. Lin TY, Wu CH, Lee WC, Chen CW, Kuo LC, Huang SL, et al: Patient outcomes following subarachnoid hemorrhage between the medical center and regional hospital: whether all patients should be transferred to medical centers. Biomed Res Int 2014:927803, 2014

25. Lindekleiv H, Mathiesen EB, Førde OH, Wilsgaard T, Ingebrigtsen T: Hospital volume and 1-year mortality after treatment of intracranial aneurysms: a study based on patient registries in Scandinavia. J Neurosurg 123:631-637, 2015

26. McDonald JS, McDonald RJ, Fan J, Kallmes DF, Lanzino G, Cloft HJ: Comparative effectiveness of ruptured cerebral aneurysm therapies: propensity score analysis of clipping versus coiling. AJNR Am J Neuroradiol 35:164-169, 2014

27. McKinney JS, Cheng JQ, Rybinnik I, Kostis JB: Comprehensive stroke centers may be associated with improved survival in hemorrhagic stroke. J Am Heart Assoc 4:e001448, 2015

28. McNeill L, English SW, Borg N, Matta BF, Menon DK: Effects of institutional caseload of subarachnoid hemorrhage on mortality: a secondary analysis of administrative data. Stroke 44:647-652, 2013

29. Mehta T, Datta N, Patel S, Mehta K, Hussain M, Kureshi I, et al: Trends in endovascular treatment of aneurysmal subarachnoid hemorrhages. Intervent Neurol 6:236-241, 2017

30. Nakamura K: Diagnosis procedure combination database would develop nationwide clinical research in Japan. Circ J 80:2289-2290, 2016

31. O'Kelly CJ, Kulkarni AV, Austin PC, Wallace MC, Urbach D: The impact of therapeutic modality on outcomes following repair of ruptured intracranial aneurysms: an administrative data analysis. Clinical article. J Neurosurg 113:795801,2010

32. Pandey AS, Gemmete JJ, Wilson TJ, Chaudhary N, Thompson BG, Morgenstern LB, et al: High subarachnoid hemorrhage patient volume associated with lower mortality and better outcomes. Neurosurgery 77:462-470, 2015

33. Rodgers M, Jobe BA, O'Rourke RW, Sheppard B, Diggs B, Hunter JG: Case volume as a predictor of inpatient mortality after esophagectomy. Arch Surg 142:829-839, 2007

34. Rush B, Romano K, Ashkanani M, McDermid RC, Celi LA: Impact of hospital case-volume on subarachnoid hemorrhage outcomes: a nationwide analysis adjusting for hemorrhage severity. J Crit Care 37:240-243, 2017

35. Sakai N, Yoshimura S, Taki W, Hyodo A, Miyachi S, Nagai Y, et al: Recent trends in neuroendovascular therapy in Japan: Analysis of a nationwide survey-Japanese Registry of Neuroendovascular Therapy (JR-NET) 1 and 2. Neurol Med Chir (Tokyo) 54:1-8, 2014

36. Shigematsu K, Nakano H, Watanabe Y: The eye response test alone is sufficient to predict stroke outcome-reintroduction of Japan Coma Scale: a cohort study. BMJ Open 3:e002736, 2013

37. Singh V, Gress DR, Higashida RT, Dowd CF, Halbach VV, Johnston SC: The learning curve for coil embolization of unruptured intracranial aneurysms. AJNR Am J Neuroradiol 23:768-771, 2002 
38. Suzuki S, Yasunaga H, Matsui H, Fushimi K, Saito Y, Yamasoba T: Cerebral infarction after intraarterial and intravenous chemoradiotherapy for head and neck cancer: a retrospective analysis using a Japanese inpatient database. Head Neck 38:1354-1358, 2016

39. Taki W, Sakai N, Suzuki H: Determinants of poor outcome after aneurysmal subarachnoid hemorrhage when both clipping and coiling are available: Prospective Registry of Subarachnoid Aneurysms Treatment (PRESAT) in Japan. World Neurosurg 76:437-445, 2011

40. Welke KF, Barnett MJ, Sarrazin MS, Rosenthal GE: Limitations of hospital volume as a measure of quality of care for coronary artery bypass graft surgery. Ann Thorac Surg 80:2114-2119, 2005

41. Woodworth GF, Baird CJ, Garces-Ambrossi G, Tonascia J, Tamargo RJ: Inaccuracy of the administrative database: comparative analysis of two databases for the diagnosis and treatment of intracranial aneurysms. Neurosurgery 65:251-257, 2009

\section{Disclosures}

The authors report no conflict of interest concerning the materials or methods used in this study or the findings specified in this paper.

\section{Author Contributions}

Conception and design: Iihara. Acquisition of data: Ogasawara,
Kitazono, Sakai, Hashimoto, Shiokawa, Miyachi, Matsumaru, Iwama, Tominaga, Onozuka, A Nishimura, Arimura, A Kurogi, Ren, Hagihara, Arai, Miyamoto. Analysis and interpretation of data: Iihara, R Kurogi, Kada, Nakaoku, K Nishimura. Drafting the article: R Kurogi. Critically revising the article: Iihara, $\mathrm{R}$ Kurogi. Reviewed submitted version of manuscript: Iihara, $\mathrm{R}$ Kurogi, Ogasawara, Kitazono, Sakai, Hashimoto, Shiokawa, Miyachi, Matsumaru, Iwama, Tominaga, Onozuka, A Nishimura, Arimura, A Kurogi, Ren, Hagihara, Arai, Miyamoto. Approved the final version of the manuscript on behalf of all authors: Iihara. Statistical analysis: Kada, Nakaoku, K Nishimura. Study supervision: Iihara.

\section{Supplemental Information}

Online-Only Content

Supplemental material is available with the online version of the article.

Supplementary eAppendices 1-8. https://thejns.org/doi/suppl/ 10.3171/2019.12.JNS192584.

\section{Correspondence}

Koji Iihara: Graduate School of Medical Sciences, Kyushu University, Fukuoka, Japan. kiihara@ns.med.kyushu-u.ac.jp. 